\title{
Prevalência de mordida aberta e fatores associados em pré-escolares de Salvador-BA em 2019
}

\author{
Prevalence of open bite and associated factors in preschoolers from Salvador-BA \\ in 2019
}

\author{
Amanda Araújo de CARVALHOa (D), Tatiana Frederico de ALMEIDA ${ }^{a}$ (D), \\ Maria Cristina Teixeira CANGUSSU ${ }^{a *}$ (1) \\ aUFBA - Universidade Federal da Bahia, Salvador, BA, Brasil
}

Como citar: Carvalho AA, Almeida TF, Cangussu MCT. Prevalência de mordida aberta e fatores associados em pré-escolares de Salvador-BA em 2019. Rev Odontol UNESP. 2020;49:e20200068. https://doi.org/10.1590/1807-2577.06820

\begin{abstract}
Resumo
Introdução: A mordida aberta anterior é um dos tipos de má-oclusão mais frequentes em pré-escolares, sendo considerada um dos problemas oclusais mais difíceis de tratar, principalmente por sua etiologia multifatorial e sua íntima relação com hábitos bucais deletérios. Objetivo: Identificar a prevalência de mordida aberta anterior nos pré-escolares de Salvador-BA, bem como fatores potencialmente associados à mesma. Material e método: Trata-se de um estudo de corte transversal em 1.577 crianças em idade pré-escolar (36 a 71 meses), que frequentavam creches públicas municipais de Salvador-BA. Resultado: A média de idade das crianças foi aproximadamente 54 meses. A maioria pertencia ao sexo masculino (50,29\%). A população de estudo foi predominantemente composta por negros e pardos (92,02\%). A prevalência de má-oclusão foi de 40,46\% e, destes, $14,02 \%$ possuíam mordida aberta. Com relação à escolaridade, a maior parte das mães (55,86\%) e dos pais (50,08\%) foi igual ou superior ao Ensino Fundamental II Completo. A sucção digital foi comum em 6,73\% das crianças e 10,39\% faziam sucção de chupeta. Foram identificados, como fatores de proteção à mordida aberta, a escolaridade do pai maior ou igual ao Ensino Fundamental II completo $(\mathrm{RP}=0,62 \mathrm{IC}=0,41-0,95)$ e a onicofagia $(\mathrm{RP}=0,34 \mathrm{IC}=0,21-0,56)$. Como fatores de risco, identificaram-se o uso de chupeta $(\mathrm{RP}=17,98$ $\mathrm{IC}=10,91-29,62)$ e a sucção digital $(\mathrm{RP}=11,04 \mathrm{IC}=6,0-20,32)$. Conclusão: Medidas educativas direcionadas aos pais e responsáveis se fazem essenciais para a prevenção do desenvolvimento de hábitos deletérios nos préescolares. Outros estudos são necessários a fim de aprofundar o entendimento sobre os fatores de proteção identificados neste estudo.
\end{abstract}

Descritores: Mordida aberta; pré-escolar; epidemiologia.

\begin{abstract}
Introduction: The previous open bite is one of the most frequent types of malocclusions in preschoolers, being considered one of the most difficult occlusal problems to treat, mainly due to its multifactorial etiology and its intimate relationship with deleterious oral habits. Objective: Identify the prevalence of anterior open bite in preschoolers in Salvador-BA as well as factors potentially associated with it. Material and method: This is a crosssectional study of 1577 preschool children (36 to 71 months) who attended municipal public daycare centers in Salvador-BA. Result: The children's average age was approximately 54 months. Most were male (50.29\%). The study population was predominantly composed of blacks and browns $(92.02 \%)$. The prevalence of malocclusion was $40.46 \%$, of which $14.02 \%$ of these children had an open bite. Regarding schooling, most mothers (55.86\%) and fathers (50.08\%) were equal to or higher than complete primary school. The digital sucking was common in 6.73\% of the children and $10.39 \%$ used pacifiers. As protective factors for open bite were found the father's education level $\geq 1$ st complete degree $(\mathrm{PR}=0.62 \mathrm{CI}=0.41-0.95)$ and nail-biting $(\mathrm{PR}=0.34 \mathrm{CI}=0.21-0.56)$. As risk factors were found the use of pacifiers $(\mathrm{PR}=17.98 \mathrm{CI}=10.91-29.62)$ and digital suction $(\mathrm{PR}=11.04 \mathrm{CI}=6.0-20.32)$. Conclusion: Educational measures aimed at parents and guardians are essential to prevent the development of harmful habits in preschoolers. Other studies are needed for in order to deepen the understanding of the identified protection factors in this study.
\end{abstract}

Descriptors: Open bite; preschool; epidemiology. 


\section{INTRODUÇÃO}

Entende-se a má-oclusão como um desarranjo no equilíbrio dental e/ou esquelético em posição de repouso e durante a mastigação e/ou a fonação. Tem consequências funcionais, estéticas e psicológicas, e trata-se de um problema de saúde pública quando em grande magnitude ou gravidade, e frequentemente acomete crianças em idade pré-escolar ${ }^{1}$. Fatores genéticos, ambientais e sociais estão relacionados com a etiologia da mesma ${ }^{2}$.

A mordida aberta anterior é um dos tipos de má-oclusão mais frequentes em pré-escolares ${ }^{3}$. Ela consiste num valor negativo para o trespasse vertical entre as incisais dos dentes da arcada superior e da arcada inferior ${ }^{4}$, e é considerada um dos problemas oclusais mais difíceis de tratar, principalmente por sua etiologia multifatorial e sua íntima relação com hábitos bucais deletérios ${ }^{4,5}$.

Os resultados apresentados no SB Brasil 2010 trazem uma prevalência de mordida aberta aos 5 anos de 12,1\%. Esse valor possui variações entre regiões do Brasil e, na literatura, esta prevalência varia de $8 \%$ a $21 \% 0^{1,3,6-9}$.

Os hábitos bucais de sucção não nutritiva, como o uso de chupetas e a sucção digital, são os fatores etiológicos mais comumente associados com a prevalência da mordida aberta anterior. Estes podem resultar em alterações neuromusculares e também interferir no desenvolvimento craniofacial ${ }^{3,10,11}$. Acredita-se que a criança que realiza sucção de chupeta de quatro a seis horas diariamente poderá ter movimentação dental significativa. $O$ ato da sucção tem um papel psicológico muito significante para crianças, cujas demandas emocionais são supridas ${ }^{12}$. Ademais, existe a hipótese de que a mordida aberta pode favorecer o desenvolvimento de outros hábitos prejudiciais, como a superposição lingual ${ }^{13}$.

Achados em literatura sugerem que hábitos parafuncionais, como a onicofagia, podem se relacionar com o aparecimento de más-oclusões e complicações musculares e articulares ${ }^{14}$. Outros hábitos, como a respiração bucal, e a função ou o tamanho anormal da língua também podem atuar como fatores etiológicos e gerar a mordida aberta 4 .

Para além disso, fatores socioeconômicos podem exercer influência no desenvolvimento desse tipo de má-oclusão, com o favorecimento na adoção de hábitos de sucção deletérios ${ }^{3}$. Contudo, relatos controversos a respeito da influência dos fatores socioeconômicos coexistem em literatura.

Apesar de ser um hábito de sucção, nesse caso nutritiva, a amamentação viabiliza o correto desenvolvimento craniofacial, tanto dentário como neuromuscular, ao estimular corretamente os tecidos. Isso faz do hábito um potencial fator de proteção para o desenvolvimento da mordida aberta ${ }^{4}$.

Acredita-se na tendência à autocorreção da mordida aberta anterior após a transição da dentição decídua para a permanente ${ }^{1,5}$. Esta é favorecida a partir do momento em que o hábito que a causou é removido. Contudo, quanto mais tardio o abandono do hábito, mais complexa se torna a correção $0^{4,13}$. Salienta-se que a gravidade da má-oclusão se relaciona estreitamente com a tríade de Graber, em que duração, intensidade e frequência do hábito deletério interagem ${ }^{13}$.

Neste sentido, o objetivo deste estudo foi identificar a prevalência de mordida aberta anterior nos pré-escolares de Salvador-BA, bem como fatores potencialmente associados à mesma.

\section{METODOLOGIA}

Desenvolveu-se um estudo de corte transversal em 1.577 crianças na faixa etária pré-escolar (36 a 71 meses), que frequentavam creches públicas municipais de Salvador-BA. Analisou-se a presença de agravos bucais, entre os quais a má-oclusão. A coleta ocorreu com a participação de oito pessoas - professoras e estudantes de graduação da Faculdade de Odontologia da 
Universidade Federal da Bahia (FOUFBA), devidamente treinados e calibrados, e foi realizada de setembro de 2018 a fevereiro de 2019, por meio de entrevista individual dos pais/ responsáveis a respeito de aspectos sociodemográficos e comportamentais da família e da criança, e de exame bucal das crianças, com o auxílio de espátula de madeira e luz natural, e os devidos equipamentos de proteção individual. Todos os responsáveis autorizaram a participação na pesquisa por meio da assinatura do Termo de Consentimento Livre e Esclarecido (TCLE).

Do questionário e exame bucal, as variáveis utilizadas neste estudo foram (Quadro 1):

Quadro 1. Classificação das variáveis analisadas no questionário aplicado aos responsáveis e no exame bucal das crianças Salvador-BA, 2018-2019

\begin{tabular}{|c|c|}
\hline VARIÁVEIS SOCIODEMOGRÁFICAS & CATEGORIZAÇÃo \\
\hline Idade & Em meses \\
\hline Sexo & 0-Masculino 1-Feminino \\
\hline Cor da pele & 1-Amarelo 2-Branco 3-Negro 4-Pardo 5-Indígena 9-Sem registro \\
\hline Escolaridade & $\begin{array}{l}\text { 0-Analfabeto 1-Alfabetizado 2-Fundamental incompleto 3- } \\
\text { Fundamental Completo 4-Ensino Médio incompleto 5- Ensino } \\
\text { Médio completo 6-Superior incompleto 7-Superior completo 8- } \\
\text { Pós-graduação 9-Não Sabe }\end{array}$ \\
\hline Com quantas pessoas a criança mora & Quantidade \\
\hline Quantos cômodos tem a casa & Quantidade \\
\hline Como é a casa onde a criança mora & 1-Alvenaria 2-Barraco 3-Apartamento 9-Sem registro \\
\hline Gestação & 1-Planejada 2-Casual 9-Sem registro \\
\hline Pré-natal & 0-Não 1-Sim 9-Sem Registro \\
\hline Durante a gestação, a mãe teve alguma doença & 0-Não 1-Sim 9-Sem Registro (Se sim, especifica-se por extenso) \\
\hline Em que local a criança nasceu & 1-Casa 2-Hospital público 3-Hospital particular 9-Sem Registro \\
\hline Tipo de parto & 1-Normal 2-Cesáreo 3-Fórceps 9-Sem Registro \\
\hline Tempo de nascimento & 1-A termo 2-Prematura 3-Pós-termo 9-Sem Registro \\
\hline COMPORTAMENTAIS & CATEGORIZAÇÃO \\
\hline A criança chorou ao nascer & 0-Não 1-Sim 9-Sem registro \\
\hline A criança ficou roxa & 0-Não 1-Sim 9-Sem registro \\
\hline A criança precisou de internação & 0-Não 1-Sim 9-Sem registro (Se sim, especifica-se o motivo) \\
\hline Até que idade foi amamentada & Em meses \\
\hline A criança range os dentes enquanto dorme & 0-Não 1-Sim 9-Sem registro \\
\hline A criança chupa chupeta & 0-Não 1-Sim 9-Sem registro \\
\hline A criança chupa dedo & 0-Não 1-Sim 9-Sem registro \\
\hline A criança morde objetos & 0-Não 1-Sim 9-Sem registro \\
\hline Alimentação equilibrada & 0-Não 1-Sim 9-Sem registro \\
\hline A criança tem alguma doença & 0-Não 1-Sim 9-Sem registro (Se sim, especifica-se a doença) \\
\hline VARIÁVEIS EM EXAME BUCAL & CATEGORIZAÇÃO \\
\hline Má-oclusão & 0-Normal 1-Leve 2-Moderada 3-Severa 9-Sem informação \\
\hline Sobremordida & 0-Normal 1-Reduzida 2-Aberta 3-Profunda 9-Sem informação \\
\hline
\end{tabular}

Elaboração própria.

A má-oclusão também foi analisada, além da mordida aberta anterior. Considerou-se, para este estudo, a variável dependente presença de mordida aberta anterior.

Procedeu-se à digitação das informações coletadas em um banco de dados no Excel e, para análise estatística, utilizou-se o programa MINITAB14. Realizaram-se análises descritivas das variáveis categóricas e contínuas, assim como uma análise exploratória em busca de potenciais fatores associados à mordida aberta.

Realizou-se o teste do qui-quadrado, com um nível de significância de 5\%, com o propósito de identificar variáveis associadas à mordida aberta anterior. Após isso, realizou-se uma análise exploratória bi e multivariada com a finalidade de estabelecer as variáveis de risco ou proteção, e a 
razão de risco para a mordida-aberta em crianças na faixa etária de 36-71 meses. Consideraram-se relevantes as variáveis com nível de significância inferior a 0,20 no modelo bivariado.

A análise de regressão logística multivariada foi realizada através da técnica stepwise, segundo o modelo de regressão de Poison, com intervalo de confiança de 95\%.

\section{RESULTADO}

Participaram do estudo 1.577 crianças em idade pré-escolar, com média de idade de aproximadamente 54 meses e desvio padrão de 10,8 meses. A maioria pertencia ao sexo masculino (50,29\%). A população de estudo foi predominantemente composta por negros e pardos (92,02\%). A maioria dos pais (52,77\%) tinham até 35 anos e $54,70 \%$ das mães tinham até 32 anos. Com relação à escolaridade, a maior parte das mães $(55,86 \%)$ e pais $(50,08 \%)$ apresentou formação igual ou superior ao Primeiro Grau completo (Tabela 1).

Tratando-se da moradia, $79,60 \%$ das crianças morvaam em casa de alvenaria ou barraco, e $76,94 \%$ delas moravam em casas secas. Dentre os pré-escolares do estudo, 77,68\% conviviam com até quatro pessoas e 75,87\% habitavam em casas com até quatro cômodos; $62,40 \%$ dos préescolares nasceram de gestações não planejadas e apenas 3,73\% do total de mães não realizou prénatal. A maioria das mães (85\%) não apresentou alguma doença durante a gestação, realizando o parto normal $(57,3 \%)$ em tempo adequado de nascimento $(80,34 \%)$. Ao nascer, $89,69 \%$ das crianças choraram, 12,3\% ficaram roxas e 19,71\% precisaram de internação (Tabela 1).

Tabela 1. Caracterização da população de estudo - pré-escolares de Salvador-BA, 2018 ( $n=1.577)$

\begin{tabular}{|c|c|c|}
\hline & $\mathbf{n}$ & $\%$ \\
\hline \multicolumn{3}{|l|}{ Sexo } \\
\hline Masculino & 793 & 50,29 \\
\hline Feminino & 784 & 49,71 \\
\hline \multicolumn{3}{|l|}{ Raça } \\
\hline Branca, Amarela e Indígena & 115 & 7,98 \\
\hline Negra e Parda & 1326 & 92,02 \\
\hline \multicolumn{3}{|l|}{ Idade da criança } \\
\hline Até 54 meses & 829 & 52,57 \\
\hline Acima de 54 meses & 748 & 47,43 \\
\hline \multicolumn{3}{|l|}{ Idade da mãe } \\
\hline Até 32 anos & 792 & 54,70 \\
\hline Acima de 32 anos & 656 & 45,30 \\
\hline \multicolumn{3}{|l|}{ Idade do pai } \\
\hline Até 35 anos & 772 & 52,77 \\
\hline Acima de 35 anos & 691 & 47,23 \\
\hline \multicolumn{3}{|l|}{ Escolaridade do pai } \\
\hline Até Ensino Fundamental II incompleto & 633 & 49,92 \\
\hline A partir do Ensino Fundamental II completo & 635 & 50,08 \\
\hline \multicolumn{3}{|l|}{ Escolaridade da mãe } \\
\hline Até Ensino Fundamental II incompleto & 637 & 44,14 \\
\hline A partir do Ensino Fundamental II completo & 806 & 55,86 \\
\hline \multicolumn{3}{|l|}{ Tipo de Casa } \\
\hline Alvenaria/Barraco & 991 & 79,60 \\
\hline Apartamento & 254 & 20,40 \\
\hline \multicolumn{3}{|l|}{ Com quantas pessoas mora } \\
\hline Até 4 pessoas & 1176 & 77,68 \\
\hline Mais de 4 pessoas & 338 & 22,32 \\
\hline
\end{tabular}


Tabela 1. Continuação...

\begin{tabular}{|c|c|c|}
\hline & $\mathbf{n}$ & $\%$ \\
\hline \multicolumn{3}{|l|}{ Cômodos da casa } \\
\hline Até 4 cômodos & 1116 & 75,87 \\
\hline Mais de 4 cômodos & 355 & 24,13 \\
\hline \multicolumn{3}{|l|}{ A casa é } \\
\hline Úmida & 295 & 23,06 \\
\hline Seca & 984 & 76,94 \\
\hline \multicolumn{3}{|l|}{ Gestação } \\
\hline Planejada & 540 & 37,60 \\
\hline Não Planejada & 896 & 62,40 \\
\hline \multicolumn{3}{|l|}{ Pré-natal } \\
\hline Sim & 1419 & 96,27 \\
\hline Não & 55 & 3,73 \\
\hline \multicolumn{3}{|l|}{ Mãe teve doença na gestação } \\
\hline Sim & 224 & 15,00 \\
\hline Não & 1269 & 85,00 \\
\hline \multicolumn{3}{|l|}{ Local de Nascimento } \\
\hline Casa/Hospital particular & 271 & 17,69 \\
\hline Hospital público & 1261 & 82,31 \\
\hline \multicolumn{3}{|l|}{ Tipo de parto } \\
\hline Normal/Fórceps & 867 & 57,80 \\
\hline Cesáreo & 633 & 42,20 \\
\hline \multicolumn{3}{|l|}{ Tempo de nascimento } \\
\hline A termo/ Pós-termo & 1175 & 85,89 \\
\hline Prematura & 193 & 14,11 \\
\hline \multicolumn{3}{|l|}{ Criança chorou ao nascer } \\
\hline Sim & 1314 & 89,69 \\
\hline Não & 151 & 10,31 \\
\hline \multicolumn{3}{|l|}{ Criança ficou roxa ao nascer } \\
\hline Sim & 144 & 12,30 \\
\hline Não & 1027 & 87,70 \\
\hline \multicolumn{3}{|c|}{ Criança necessitou de internação } \\
\hline Sim & 300 & 19,71 \\
\hline Não & 1222 & 80,29 \\
\hline
\end{tabular}

Elaboração própria.

Na população de estudo, a prevalência de má-oclusão foi de 40,46\% e, dentre estes, 14,02\% possuíam mordida aberta.

No que concerne aos hábitos, a maioria das crianças foi amamentada até 12 meses de idade. A sucção digital era realizada por $6,73 \%$ dos pré-escolares e $10,39 \%$ faziam o uso de chupetas. A maioria das crianças $(69,43 \%)$ não possuía o hábito de morder objetos e $26,14 \%$ rangiam os dentes (Tabela 2).

Tabela 2. Hábitos deletérios e condições bucais em pré-escolares de Salvador-BA, 2018 ( $n=1577)$

\begin{tabular}{lcc}
\hline & $\mathbf{n}$ & $\mathbf{\%}$ \\
\hline Idade de amamentação & & \\
Até 12 meses & 810 & 56,60 \\
Acima de 12 meses & 621 & 43,40 \\
Sucção Digital & & \\
Sim & 81 & 6,73 \\
Não & 1123 & 93,27 \\
\hline
\end{tabular}


Tabela 2. Continuação...

\begin{tabular}{lcc}
\hline & $\mathbf{n}$ & \% \\
\hline Uso de Chupeta & & 10,39 \\
Sim & 154 & 89,61 \\
Não & 1328 & \\
Range os Dentes & & 26,14 \\
Sim & 377 & 73,86 \\
Não & 1065 & \\
Morde Objetos & & 30,57 \\
Sim & 372 & 69,43 \\
Não & 845 &
\end{tabular}

Elaboração própria.

Na Tabela 3, foi feita a distribuição das variáveis de interesse segundo a presença e ausência de mordida aberta nos pré-escolares. As variáveis idade da criança, escolaridade do pai, escolaridade da mãe, cômodos da casa, gestação, local de nascimento, idade de amamentação, usa chupeta, sucção digital e rói unha mostraram-se relevantes com um p-valor inferior a 0,20 e foram exploratoriamente levadas ao modelo multivariado final.

Tabela 3. Distribuição das variáveis de interesse segundo a presença de Mordida Aberta em pré-escolares de Salvador-BA, $2018(n=1577)$

\begin{tabular}{|c|c|c|c|c|c|}
\hline \multicolumn{6}{|c|}{ Mordida Aberta } \\
\hline & \multicolumn{2}{|c|}{ Ausente } & \multicolumn{2}{|c|}{ Presente } & \multirow{2}{*}{ P-valor } \\
\hline & $\mathbf{n}$ & $\%$ & $\mathbf{n}$ & $\%$ & \\
\hline \multicolumn{6}{|l|}{ Sexo } \\
\hline Masculino & 687 & 50,93 & 105 & 47,73 & 0,379 \\
\hline Feminino & 662 & 49,07 & 115 & 52,27 & \\
\hline \multicolumn{6}{|l|}{ Raça } \\
\hline Branca, Amarela e Indígena & 99 & 7,98 & 16 & 8,29 & 0,882 \\
\hline Negra e Parda & 1142 & 92,02 & 177 & 91,71 & \\
\hline \multicolumn{6}{|l|}{ Idade da criança } \\
\hline Até 54 meses & 693 & 51,37 & 134 & 60,91 & 0,009 \\
\hline Acima de 54 meses & 656 & 48,63 & 86 & 39,09 & \\
\hline \multicolumn{6}{|l|}{ Idade do pai } \\
\hline Até 35 anos & 661 & 53,05 & 107 & 51,20 & 0,619 \\
\hline Acima de 35 anos & 585 & 46,95 & 102 & 48,80 & \\
\hline \multicolumn{6}{|l|}{ Idade da mãe } \\
\hline Até 32 anos & 671 & 54,38 & 120 & 58,25 & 0,301 \\
\hline Acima de 32 anos & 563 & 45,62 & 86 & 41,75 & \\
\hline \multicolumn{6}{|l|}{ Escolaridade do pai } \\
\hline Até o Ensino Fundamental II completo & 527 & 48,66 & 102 & 57,63 & 0,027 \\
\hline A partir do Ensino Fundamental II completo & 556 & 51,34 & 75 & 42,37 & \\
\hline \multicolumn{6}{|l|}{ Escolaridade da mãe } \\
\hline Até Ensino Fundamental II completo & 531 & 43,03 & 104 & 51,74 & 0,021 \\
\hline A partir do Ensino Fundamental II completo & 703 & 56,97 & 97 & 48,26 & \\
\hline \multicolumn{6}{|l|}{ Com quantas pessoas mora } \\
\hline Até 4 pessoas & 1007 & 77,76 & 161 & 76,30 & 0,638 \\
\hline Mais de 4 pessoas & 288 & 22,24 & 50 & 22,44 & \\
\hline \multicolumn{6}{|l|}{ Cômodos da casa } \\
\hline Até 4 cômodos & 943 & 75,02 & 168 & 81,55 & 0,042 \\
\hline Acima de 4 cômodos & 314 & 24,98 & 38 & 18,45 & \\
\hline
\end{tabular}


Tabela 3. Continuação...

\begin{tabular}{|c|c|c|c|c|c|}
\hline \multicolumn{6}{|c|}{ Mordida Aberta } \\
\hline & \multicolumn{2}{|c|}{ Ausente } & \multicolumn{2}{|c|}{ Presente } & \multirow{2}{*}{ P-valor } \\
\hline & $\mathbf{n}$ & $\%$ & $\mathbf{n}$ & $\%$ & \\
\hline \multicolumn{6}{|l|}{ Tipo de casa } \\
\hline Alvenaria/Barraco & 850 & 79,29 & 137 & 83,03 & 0,266 \\
\hline Apartamento & 222 & 20,71 & 28 & 16,97 & \\
\hline \multicolumn{6}{|l|}{ A casa é } \\
\hline Úmida & 249 & 22,76 & 44 & 24,31 & 0,646 \\
\hline Seca & 845 & 77,24 & 137 & 75,69 & \\
\hline \multicolumn{6}{|l|}{ Gestação } \\
\hline Planejada & 478 & 38,77 & 57 & 29,08 & 0,009 \\
\hline Não Planejada & 755 & 61,23 & 139 & 70,92 & \\
\hline \multicolumn{6}{|l|}{ Pré Natal } \\
\hline Sim & 1218 & 96,28 & 193 & 96,02 & 0,854 \\
\hline Não & 47 & 3,72 & 8 & 3,98 & \\
\hline \multicolumn{6}{|l|}{ Doença na gestação } \\
\hline Sim & 187 & 14,63 & 37 & 17,87 & 0,227 \\
\hline Não & 1091 & 85,37 & 170 & 82,13 & \\
\hline \multicolumn{6}{|l|}{ Local de Nascimento } \\
\hline Casa/Hospital particular & 237 & 18,13 & 31 & 14,29 & 0,168 \\
\hline Hospital público & 1070 & 81,87 & 186 & 85,71 & \\
\hline \multicolumn{6}{|l|}{ Tipo de Parto } \\
\hline Normal/Fórceps & 742 & 58,01 & 123 & 57,75 & 0,942 \\
\hline Cesáreo & 537 & 41,99 & 90 & 42,25 & \\
\hline \multicolumn{6}{|l|}{ Tempo de nascimento } \\
\hline Prematuro & 161 & 13,84 & 31 & 15,74 & 0,481 \\
\hline A termo/Pós-termo & 1002 & 86,16 & 166 & 84,26 & \\
\hline \multicolumn{6}{|l|}{ A criança chorou ao nascer } \\
\hline Sim & 1127 & 89,87 & 180 & 88,24 & 0,477 \\
\hline Não & 127 & 10,13 & 24 & 11,76 & \\
\hline \multicolumn{6}{|l|}{ A criança ficou roxa } \\
\hline Sim & 119 & 11,92 & 24 & 14,46 & 0,357 \\
\hline Não & 879 & 88,08 & 142 & 85,54 & \\
\hline \multicolumn{6}{|l|}{ A criança foi internada } \\
\hline Sim & 252 & 19,38 & 44 & 20,56 & \\
\hline Não & 1048 & 80,62 & 170 & 79,44 & 0,688 \\
\hline \multicolumn{6}{|l|}{ Idade de amamentação } \\
\hline Até 12 meses & 658 & 54,07 & 149 & 72,33 & \\
\hline Acima de 12 meses & 559 & 45,93 & 57 & 27,67 & 0,00 \\
\hline \multicolumn{6}{|l|}{ Range os dentes } \\
\hline Sim & 328 & 26,62 & 48 & 23,65 & 0,371 \\
\hline Não & 904 & 73,38 & 155 & 76,35 & \\
\hline \multicolumn{6}{|l|}{ Usa chupeta } \\
\hline Sim & 65 & 94,87 & 88 & 42,72 & 0,00 \\
\hline Não & 1203 & 5,13 & 118 & 57,28 & \\
\hline \multicolumn{6}{|l|}{ Sucção digital } \\
\hline Sim & 46 & 4,51 & 35 & 20,00 & 0,00 \\
\hline Não & 975 & 95,49 & 140 & 80,00 & \\
\hline Rói unha & & & & & \\
\hline Sim & 487 & 38,80 & 43 & 21,29 & 0,00 \\
\hline Não & 768 & 61,20 & 159 & 78,71 & \\
\hline Morde objetos & & & & & \\
\hline Sim & 315 & 30,67 & 54 & 29,67 & 0,787 \\
\hline Não & 712 & 69,33 & 128 & 70,33 & \\
\hline
\end{tabular}

Elaboração própria. 
0 resultado final das variáveis associadas à mordida aberta foi: escolaridade do pai $\leq$ Ensino Fundamental II completo, usa chupeta, sucção digital e rói unha. Houve associações positivas como potenciais fatores de proteção para a não ocorrência de mordida aberta: escolaridade do pai $\geq 1^{\underline{o}}$ Grau completo com chance para o desenvolvimento dessa má-oclusão, sendo 0,38 vez menor (IC 95\% 0,41-0,95) e rói unha com chance 0,66 vez menor (IC 95\% 0,21-0,56) (P-valor $=0,00$ ). Ademais, usa chupeta apresenta risco 18 vezes maior (IC 95\% 10,91-29,62) para mordida aberta e sucção digital com risco 11 vezes maior (IC 95\% 6,0-20,32). Estas foram reconhecidas como variáveis potenciais de risco, com associação significativa com a mordida aberta (P-valor $=0,00$ ).

\section{DISCUSSÃO}

No estudo, identificou-se a prevalência de $14,02 \%$ de mordida aberta. Foi observada a associação do uso de chupeta e da sucção digital com a maior ocorrência da má-oclusão estudada. Além disso, registrou-se a associação negativa com maior escolaridade do pai e a presença de onicofagia.

Tratando-se dos hábitos de sucção não nutritiva, no presente estudo, o uso de chupetas foi identificado como um dos maiores fatores de risco para o desenvolvimento da mordida aberta. A sucção de chupeta não só está associada com a mordida aberta como pode levar ao abandono precoce da amamentação, a qual poderia, por sua vez, atuar como proteção a não adoção dos hábitos de sucção não nutritiva ${ }^{2,3,15}$. Quando sustentado, o hábito possui maior potencial danoso e para aparecimento da má-oclusão ${ }^{16} .0$ aumento no uso de chupetas pode ser relacionado com fatores socioculturais e o modo de vida moderno ${ }^{8}$.

Por sua vez, a sucção digital demonstrou ser outro fator de risco para a mordida aberta. Associadamente, esse mesmo achado é documentado pela literatura ${ }^{3}$. É entendida como o hábito mais difícil de ser abandonado se comparado com a sucção de chupeta ${ }^{11}$. Ademais, ela pode gerar anormalidades na erupção dentária e alterar o desenvolvimento esquelético, gerando também a mordida aberta ${ }^{2,15}$.

No atual estudo, o uso de chupeta demonstrou ser mais prejudicial que a sucção digital. De modo geral, independentemente do hábito, as crianças que possuem hábitos de sucção não nutritiva geralmente demonstram maior prevalência de mordida aberta ${ }^{15}$.

Ao tratar-se de potenciais fatores de proteção ao desenvolvimento da mordida aberta, a escolaridade do pai foi um fator identificado. Entende-se que a mordida aberta está associada com aspectos sociodemográficos e, mais fortemente, com aqueles que se encontram em um baixo nível econômico. Além disso, fatores sociodemográficos podem influenciar na adoção de hábitos deletérios de sucção ${ }^{3}$. Porém, alguns achados em literatura não identificaram associação significante entre a mordida aberta e o nível de educação dos pais ou o nível econômico familiar8,17. Assim, são necessários mais estudos que avaliem a associação da mordida aberta com aspectos sociodemográficos.

No que concerne à onicofagia, foi um hábito identificado como potencial fator de proteção à mordida aberta. Consiste em um dos hábitos parafuncionais mais prevalentes, sendo relacionado com o estado emocional 14,15 . Contudo, achados em literatura associam o hábito com o desenvolvimento de más-oclusões ${ }^{14}$. Sua proteção pode acontecer devido à necessidade de toque entre as incisais dos dentes anteriores com a borda da unha para o estabelecimento do hábito, inviabilizando assim a adoção de hábitos como a sucção digital e o uso da chupeta.

Para além dos achados do presente estudo, deve-se levar em consideração a influência de aspectos hereditários e genéticos no desenvolvimento da má-oclusão. A ausência de resposta no questionário aplicado pode levar à subestimação de variáveis, o que se constitui como uma limitação do estudo. Além disso, deve-se considerar que características culturais de cada região podem interferir nos hábitos da população. 
Salienta-se que medidas preventivas e consultas regulares com o cirurgião-dentista podem auxiliar no tratamento e evitar agravos ou aparecimento da mordida aberta. Os dados identificados pelo estudo poderão servir de base para o estabelecimento de medidas de intervenção em controle e prevenção da mordida aberta na faixa etária pré-escolar no município de Salvador-BA e, consequentemente, atuarão em sentido de redução da prevalência da mordida aberta em crianças.

\section{CONCLUSÃO}

Em Salvador-BA, os pré-escolares apresentam prevalência de 14,02\% de mordida aberta. Além disso, foram identificadas variáveis associadas à má-oclusão com significância estatística. Como fatores de risco, o hábito do uso de chupeta expõe o pré-escolar a um risco aproximadamente 18 vezes (IC 95\% 10,91-29,62) maior de desenvolver a mordida aberta, seguido da sucção digital, que apresenta um risco 11 vezes (IC 95\% 6,0-20,32) maior no desenvolvimento da má-oclusão estudada. Ao tratarmos dos fatores de proteção, possuir o hábito de onicofagia representa uma propensão 0,66 vez (IC 95\% 0,21-0,56) menor de desenvolver a mordida aberta, seguida da escolaridade do pai a partir do Ensino Fundamental II completo, em que a propensão para o desenvolvimento dessa má-oclusão é 0,38 vez (IC 95\% 0,41-0,95) menor. Dessa forma, medidas educativas direcionadas aos pais e responsáveis se fazem essenciais para a prevenção do desenvolvimento de hábitos deletérios nos pré-escolares. Ademais, outros estudos são necessários a fim de aprofundar o entendimento sobre os fatores de proteção identificados.

\section{REFERÊNCIAS}

1. Bauman JM, Souza JGS, Bauman CD, Flório FM. Padrão epidemiológico da má oclusão em pré-escolares brasileiros. Cien Saude Colet. 2018 Nov;23(11):3861-8. http://dx.doi.org/10.1590/1413812320182311.24722016. PMid:30427456.

2. Paolantonio EG, Ludovici N, Saccomanno S, La Torre G, Grippaudo C. Association between oral habits, mouth breathing and malocclusion in Italian preschoolers. Eur J Paediatr Dent. 2019 Sep;20(3):204-8. http://dx.doi.org/10.23804/ejpd.2019.20.03.07. PMid:31489819.

3. Gomes MC, Neves ETB, Perazzo MF, Martins CC, Paiva SM, Granville-Garcia AF. Association between psychological factors, sociodemographic conditions, oral habits and anterior open bite in five-year-old children. Acta Odontol Scand. 2018 Nov;76(8):553-8. http://dx.doi.org/10.1080/00016357.2018.1472294. PMid:29764280.

4. Nakao TH, Bertoz FA, Oliveira DTN, Bertoz APM, Bigliazzi R. Hábitos bucais como fatores de risco para a mordida abeta anterior: uma revisão de literatura. Rev Odontol Araçatuba. 2016;37(2):46-51.

5. Rosa M, Quinzi V, Marzo G. Paediatric orthodontics part 1: anterior open bite in the mixed dentition. Eur J Paediatr Dent. 2019 Mar;20(1):80-2. http://dx.doi.org/10.23804/ejpd.2019.20.01.15. PMid:30919650.

6. Brasil. Ministério da Saúde. Projeto SB Brasil 2010: Pesquisa Nacional de Saúde Bucal: resultados principais. Brasília, DF; 2012.

7. Vilain CT, Mendes L, Simões PW, Vanni PJJ, Ceretta LB, Ceretta RA, et al. Prevalência de maloclusão em crianças de 05 anos de idade em um município catarinense. Rev Odontol Univ Cid São Paulo. 2016 SetDez;28(3):210-22.

8. Miotto MHMB, Rossi F. Júnior, Barcellos LA, Campos DMKS. Prevalência da mordida aberta anterior em crianças de 3 a 5 anos. Arq Odontol. 2016;52(2):111-6. 
9. Sousa RV, Pinto-Monteiro AKA, Martins CC, Granville-Garcia AF, Paiva SM. Malocclusion and socioeconomic indicators in primary dentition. Braz Oral Res. 2014;28(1):54-60. http://dx.doi.org/10.1590/S1806-83242013005000032. PMid:25000602.

10. Ling HTB, Sum FHKMH, Zhang L, Yeung CPW, Li KY, Wong HM, et al. The association between nutritive, non-nutritive sucking habits and primary dental occlusion. BMC Oral Health. 2018 Aug;18(1):145. http://dx.doi.org/10.1186/s12903-018-0610-7. PMid:30134878.

11. Silvestrini-Biavati A, Salamone S, Silvestrini-Biavati F, Agostino P, Ugolini A. Anterior open-bite and sucking habits in Italian preschool children. Eur J Paediatr Dent. 2016 Mar;17(1):43-6. PMid:26949238.

12. Garbin CAS, Garbin AJI, Martins RJ, Souza NP, Moimaz SAS. Prevalência de hábitos de sucção não nutritivos em pré-escolares e a percepção dos pais sobre sua relação com maloclusões. Cien Saude Colet. 2014 Feb;19(2):553-8. http://dx.doi.org/10.1590/1413-81232014192.23212012. PMid:24863831.

13. Nascimento MHA, Araújo TM, Machado AW. Severe anterior open bite during mixed dentition treated with palatal spurs. J Clin Pediatr Dent. 2016;40(3):247-50. http://dx.doi.org/10.17796/1053-462840.3.247. PMid:27472574.

14. Camargo TN, Brum SC, Barbosa CCN, Chaves ES, Oliveira RS. Avaliação de impeditivo resinoso para o hábito de onicofagia. Revista Pró-UniverSUS. 2015 Jul-Dez;6(3):49-53.

15. Lopes TSP, Lima CCB, Silva RNC, Moura LFAD, Lima MDM, Lima MCMP. Association between duration of breastfeeding and malocclusion in primary dentition in Brazil. J Dent Child (Chic). 2019 Jan;86(1):17-23. PMid:30992097.

16. Grippaudo C, Paolantonio EG, Antonini G, Saulle R, La Torre G, Deli R. Association between oral habits, mouth breathing and malocclusion. Acta Otorhinolaryngol Ital. 2016 Oct;36(5):386-94. http://dx.doi.org/10.14639/0392-100X-770. PMid:27958599.

17. Zhou Z, Liu F, Shen S, Shang L, Shang L, Wang X. Prevalence of and factors affecting malocclusion in primary dentition among children in Xi'an, China. BMC Oral Health. 2016;16(1):91. http://dx.doi.org/10.1186/s12903-016-0285-x. PMid:27590299.

\section{CONFLITOS DE INTERESSE}

Os autores declaram não haver conflitos de interesse.

\section{*AUTOR PARA CORRESPONDÊNCIA}

Maria Cristina Teixeira Cangussu, UFBA - Universidade Federal da Bahia, Rua Araújo Pinho, 62, Canela, 6oandar, 41110-150 Salvador - BA, Brasil, e-mail: cangussu@ufba.br

Recebido: Outubro 7, 2020

Aprovado: Novembro 18, 2020 